\title{
Identification, Characterization, and Functional Analysis of Chitin Synthase Genes in Glyphodes pyloalis Walker (Lepidoptera: Pyralidae)
}

\author{
Zuo-Min Shao ${ }^{1}$, Yi-Jiangcheng Li ${ }^{1}$, Jian-Hao Ding ${ }^{1}$, Zhi-Xiang Liu ${ }^{1}$, Xiao-Rui Zhang ${ }^{1}$,

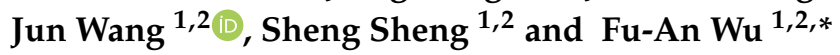 \\ 1 Jiangsu Key Laboratory of Sericultural Biology and Biotechnology, School of Biotechnology, \\ Jiangsu University of Science and Technology, Zhenjiang 212018, China; shaozuomin23@163.com (Z.-M.S.); \\ 192310019@stu.just.edu.cn (Y.-J.L.); lostone1@126.com (J.-H.D.); 18852864112@163.com (Z.-X.L.); \\ zxr1131286377@sina.com (X.-R.Z.); wangjun@just.edu.cn (J.W.); parasitoids@163.com (S.S.) \\ 2 Key Laboratory of Silkworm and Mulberry Genetic Improvement, Ministry of Agriculture and Rural Affairs, \\ Sericultural Research Institute, Chinese Academy of Agricultural Science, Zhenjiang 212018, China \\ * Correspondence: fuword@163.com
}

Received: 14 June 2020; Accepted: 29 June 2020; Published: 30 June 2020

\begin{abstract}
Glyphodes pyloalis Walker (G. pyloalis) causes significant damage to mulberry every year, and we currently lack effective and environmentally friendly ways to control the pest. Chitin synthase (CHS) is a critical regulatory enzyme related to chitin biosynthesis, which plays a vital role in the growth and development of insects. The function of CHS in G. pyloalis, however, has not been studied. In this study, two chitin synthase genes (GpCHSA and GpCHSB) were screened from our previously created transcriptome database. The complete coding sequences of the two genes are 5,955 bp and 5,896 bp, respectively. Expression of GpCHSA and GpCHSB could be detected throughout all developmental stages. Relatively high expression levels of GpCHSA occurred in the head and integument and GpCHSB was most highly expressed in the midgut. Moreover, silencing of GpCHSA and $G p C H S B$ using dsRNA reduced expression of downstream chitin metabolism pathway genes and resulted in abnormal development and wings stretching, but did not affect normal pupating of larvae. Furthermore, the inhibitor of chitin synthesis diflubenzuron (DFB) was used to further validate the RNAi result. DFB treatment significantly improved expression of GpCHSA, except GpCHSB, and their downstream genes, and also effected G. Pyloali molting at $48 \mathrm{~h}(62 \%$ mortality rate) and $72 \mathrm{~h}$ ( $90 \%$ mortality rate), respectively. These results show that GpCHSA and GpCHSB play critical roles in the development and wing stretching in G. pyloalis adults, indicating that the genes are attractive potential pest control targets.
\end{abstract}

Keywords: Glyphodes pyloalis Walker; chitin synthase; DFB; RNA interference; pest control

\section{Introduction}

Glyphodes pyloalis Walker is an important mulberry pest which is widely distributed throughout major mulberry growing areas of China, India, Korea, Japan, Pakistan, and Burma. This insect damages sericulture not only by feeding on mulberry but also by transmitting viruses to the silkworm [1]. In recent years, the outbreak of $G$. pyloalis has resulted in severe losses to local mulberry farmers. Currently, using conventional insecticides is the principal means to control G. pyloalis populations. However, the heavy use of insecticides in mulberry growing regions has caused silkworm poisoning and environmental pollution, as well as increased G. pyloalis resistance to a variety of chemical insecticides including organophosphates, pyrethroids, and carbamates [2]. Therefore, there is an urgent need for environmentally-friendly methods to control G. pyloalis. 
Chitin is the second most abundant natural polymer (after cellulose), and it is a polymer of $N$-acetyl- $\beta$-D-glucosamine (GlcNAc). It is widely distributed throughout fungus, arthropod, nematode, and marine organism [3-6]. In insects, chitin is an essential component of the cuticle and peritrophic matrix (PM), which plays an important role in keeping insect shape and protecting itself from external stresses, respectively. Periodic molting is the process that involves the shedding and replacing of the thick insect exoskeleton, and is an essential step that occurs during insect growing development. Throughout the process, part of the old cuticle is digested, and then new chitin is synthesized. Chitin metabolism is regulated by three main enzymes, chitin synthase (CHS), chitin deacetylase (CDA), and chitinase (CHT) $[7,8]$. CHS was first identified in yeast and fungi $[9,10]$ and contains two conserved motifs, EDR and QRRRW [11], which are found in CHS of all chitin-synthesizing organisms [12]. It is one kind of transmembrane protein from a large glycosyltransferases family, and is involved in catalyzing the polymerization of UDP- $N$-acetylglucosamine (UDP-GlcNAc) from chitin. $\mathrm{CHS}$ have been found existed in many insect species [13], including Drosophila melanogaster (D. melanogaster)[14], Choristoneura fumiferana [15], Anopheles gambiae (A. gambiae)[16], Locusta migratoria manilensis [17], Tribolium castaneum (T. castaneum)[18], Plutella xylostella [19], and Manduca sexta [20]. Currently, two kinds of CHS genes in insects have been identified, CHSA and CHSB (also called CHS1 and CHS2). CHSA is mainly reported in the cuticle and tracheae, and CHSB is mainly reported in midgut epithelial cells and associated with the formation of the PM. Therefore, CHS plays an important role in insect growing development by regulating molting and cuticle regeneration. Disruption of chitin synthesis has been extensively recognized as a promising means to control insect populations [12,21].

Diflubenzuron (DFB) [1-(4-chlorophenyl)-3-(2.6-diflubenzoyl)urea] is the first benzoylphenylurea (BPU) chitin synthesis inhibitor that acts by controlling chitin synthesis in various pest species [21,22]. Exposure of Panonychus citri to DFB causes abortive molting and high rates of larvae mortality [23]. Treatment of Anopheles quadrimaculatus (A. quadrimaculatus) with DFB significantly reduces chitin content. Moreover, the expression of $A q C H S 1$ was significantly upregulated after exposure to DFB in A. quadrimaculatus [12]. RNA interference (RNAi) is a useful tool used to analyze gene function in different organisms by delivering gene-specific, double-stranded RNA (dsRNA). It is also known as a promising approach for pest control [24]. RNAi has been reported to assess the function of $C H S$ in many insect species. Silencing of $A$. gambiae CHS1 and CHS2 via chitosan/AgCHS dsRNA-based nanoparticles feeding resulted in the downregulation of both genes and reduced chitin accumulation. The treatment lead to enhanced larval susceptibility to diflubenzuron [25]. Silencing of Diaphorina citri CHS caused malformed phenotypes, increased mortality, and decreased molting rates [26]. The function of Acyrthosiphon pisum CHS was studied by injecting insects with dsRNA, and results revealed that CHS plays an important function in embryonic development and nymphal growth [24]. RNAi was used to target Toxoptera citricida CHS via feeding fourth instar with dsRNA, which rendered insects incapable of molting [27]. However, the function of CHS in G. pyloalis remains unclear. Thus, clarification of the function of $\mathrm{CHS}$ genes in G. pyloalis may contribute to identify candidate molecular targets for control of the pest.

In this study, GpCHSA and GpCHSB were identified from previous transcriptome data [28]. The sequences of the genes were analyzed using bioinformatics, and expression patterns of the genes in different tissues and developmental stages were analyzed using RT-qPCR. The function of each gene was studied using DFB and dsRNA treatment. Our study preliminarily clarifies the function of GpCHSA and GpCHSB in the chitin metabolism pathway and may facilitate the finding of new tools for managing G. pyloalis.

\section{Results}

\subsection{Characterization of the GpCHSA and GpCHSB Sequences}

GpCHSA and GpCHSB cDNA sequences have been uploaded to NCBI GenBank, and accession numbers are MN915086 and MN915087, respectively. The full-length of GpCHSA is 5,955 bp, which 
contains a 4,695 bp open reading frame (ORF) that encodes a 1,564 amino acid protein with a predicted molecular mass (MW) of $178.52 \mathrm{kDa}$ and isoelectric point (pI) of 6.54. Full-length of GpCHSB is 5,896 $\mathrm{bp}$, and contains a 4,590 bp ORF that encodes a protein with 1,529 amino acid residues. The protein has a predicted MW of $174.99 \mathrm{kDa}$ and $\mathrm{pI}$ of 6.27. The putative catalytic domain of GpCHSs included two representative motifs EDR and QRRRW (Figure 1). The catalytic functional domain of chitin synthases shared $94.02 \%$ amino acid identity in different Lepidoptera insects based on the multiple sequence alignment (Figure 1). Moreover, the full structure of the GpCHSA protein consists of 13 transmembrane regions, one low complexity region, and 2 coiled-coil regions (Figure 2A). GpCHSB protein also contains 13 transmembrane regions, two low complexity regions, and one coiled coil regions (Figure 2B). Detailed sequence information of GpCHSA and GpCHSB are showed in Figure S1.

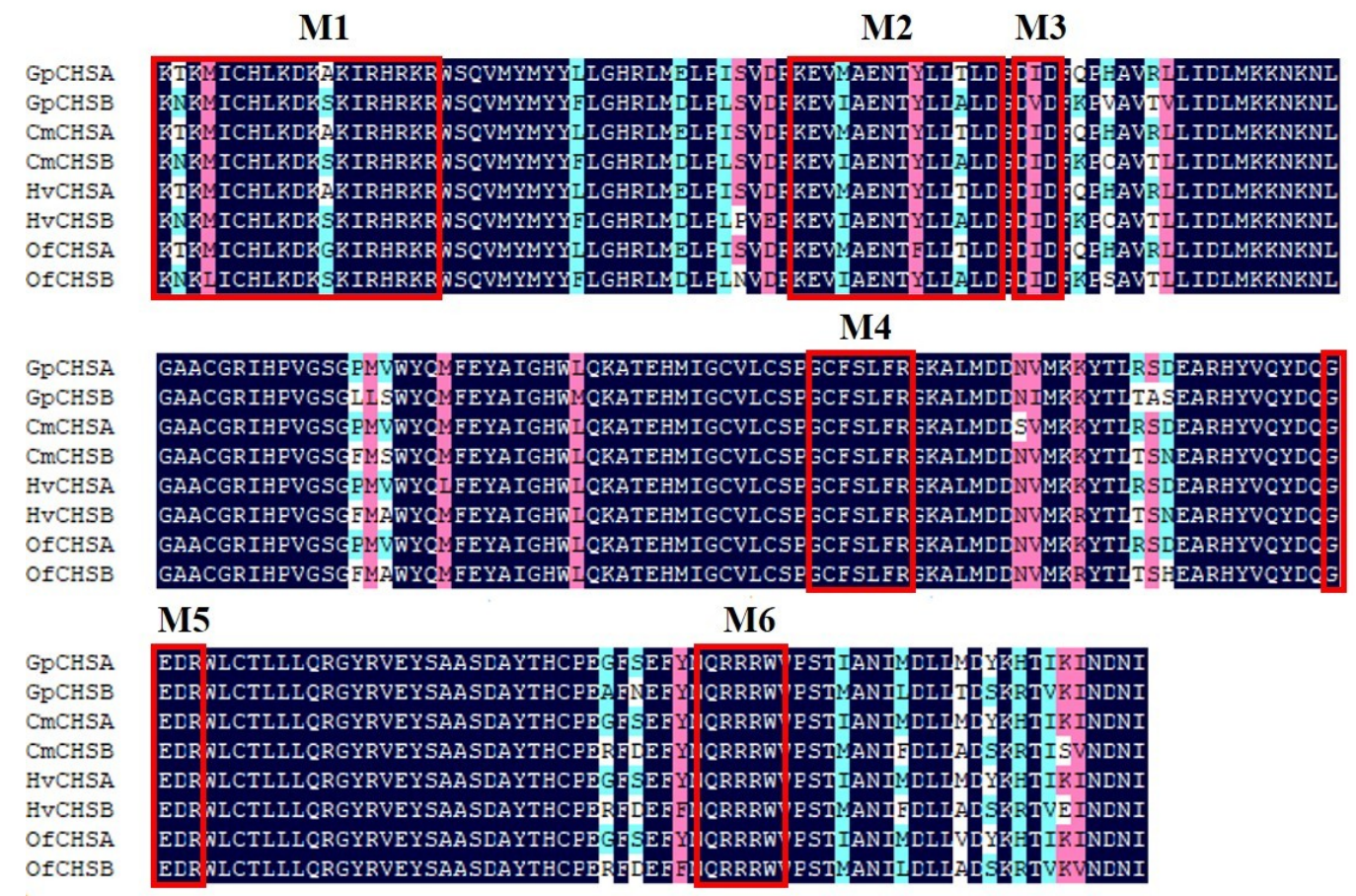

Figure 1. The analysis of putative catalytic functional domains of chitin synthases (CHSs) among different Lepidoptera insects based on the multiple sequence alignment. Dark blue represents identical amino acids. Pink and aqua represent positively charged amino acids. Non-conserved positions have a white background. Six characteristic motifs M1-6 of highly conserved regions of glycosyltransferases (family 2) enzymes were shown in red boxes [22,29]. Cm, Cnaphalocrocis medinalis, CmCHSA, AJG44538.1, CmCHSB, AJG44539.1; Hv, Heortia vitessoides, HvCHSA, AZQ19982.1, HvCHSB, AZQ19981.1; Of, Ostrinia furnacalis, OfCHSA, ACF53745.1, OfCHSB, ABB97082.1. 


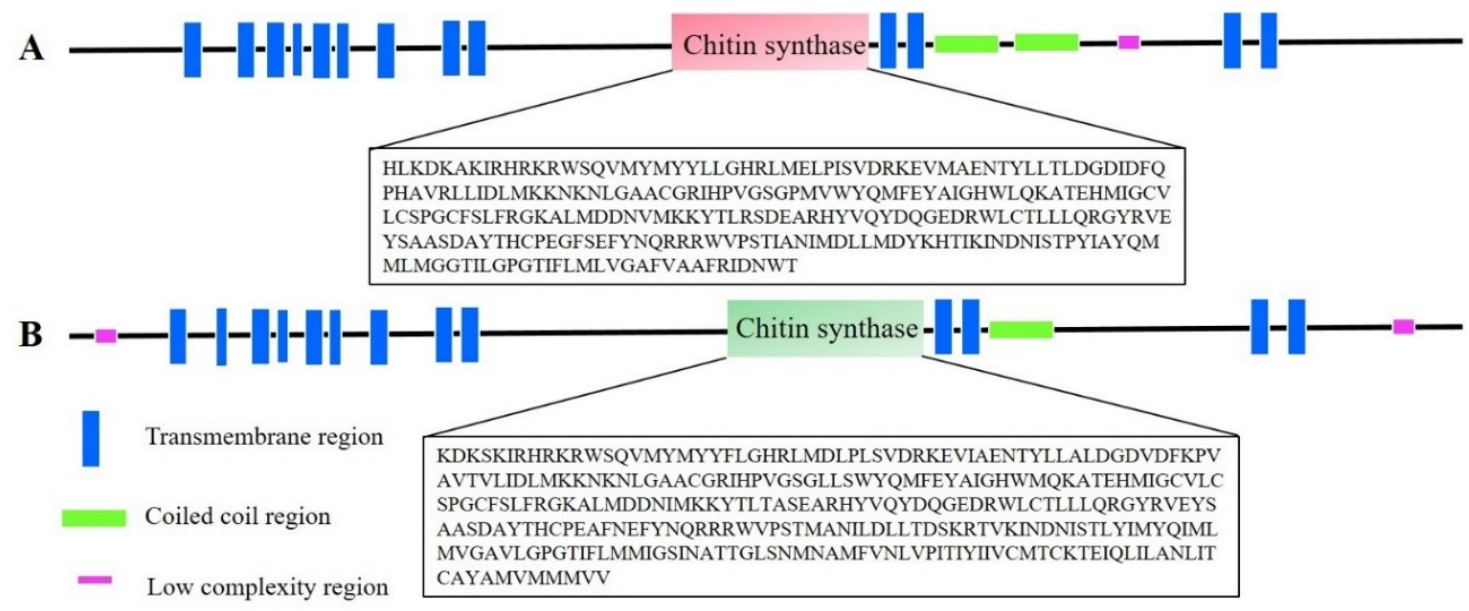

Figure 2. The predicted conserved functional domains of GpCHSA and GpCHSB. Different colors are used to mark predicted domains. The conserved functional domain of GpCHSA consists of 261 amino acids (A), and GpCHSB has 302 amino acids (B). Conserved sequences were identified using SMART (http://smart.embl-heidelberg.de/) website and indicated in boxed.

To get the phylogenetic tree between CHSA and CHSB in different species, MEGA-X software was used. A total of 33 CHSs from 20 species were grouped into two phylogenetic groups, CHSA and CHSB, indicating that some kinds of difference between of them do exist. Moreover, the result showed that both GpCHSA and GpCHSB have a close relationship with Cnaphalocrocis medinalis CHSA and CHSB, respectively (Figure 3).

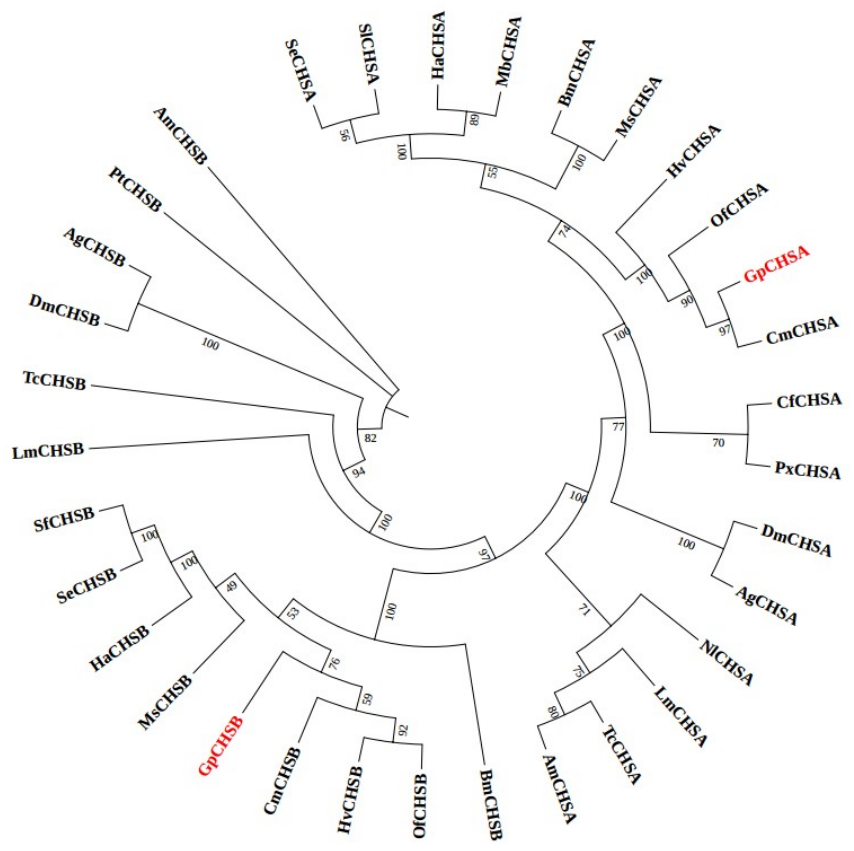

Figure 3. Phylogenetic relationship analysis of CHSs in different identified insect species using the neighbor-joining method with 1,000 bootstraps. Bm, Bombyx mori; Cf, Choristoneura fumiferana; $\mathrm{Cm}$, Cnaphalocrocis medinalis; Dm, Drosophila melanogaster; Ha, Helicoverpa armigera; Lm, Locusta migratoria; Ms, Manduca sexta; Px, Plutella xylostella; Se, Spodoptera exigua; Tc, Tribolium castaneum; Of, Ostrinia furnacalis; Sf, Spodoptera frugiperda; Hv, Heortia vitessoides; Ag, Anopheles gambiae; Pt, Parasteatoda tepidariorum; Am, Apis mellifera; Mb, Mamestra brassicae; Nl, Nilaparvata lugens; Sl, Spodoptera litura. 


\subsection{Spatio-Temporal Expression Patterns of GpCHSA and GpCHSB}

To preliminarily get the specific biological function of GpCHSA and GpCHSB, relative expression levels of both genes were detected in different developmental stages and tissues of fifth instar larvae using RT-qPCR. The expression levels showed that both genes expressed throughout all of developmental stages, which indicated that GpCHSA and GpCHSB were involved in chitin metabolism throughout G. pyloalis whole life. Moreover, GpCHSA expression was determined to be the highest in the pupa, and GpCHSB expression peaked at the larval stage (Figure 4). Moreover, the expression levels of $G p C H S A$ in the head and integument were 272 and 108 fold greater than that in the midgut, respectively. The greatest expression level of GpCHSB was in the midgut that was 727 times higher than that in the integument (Figure 4). Therefore, we suspected that GpCHSA might participate in the integument chitin synthesis pathway, while GpCHSB is more likely to be involved in the midgut chitin synthesis pathway.
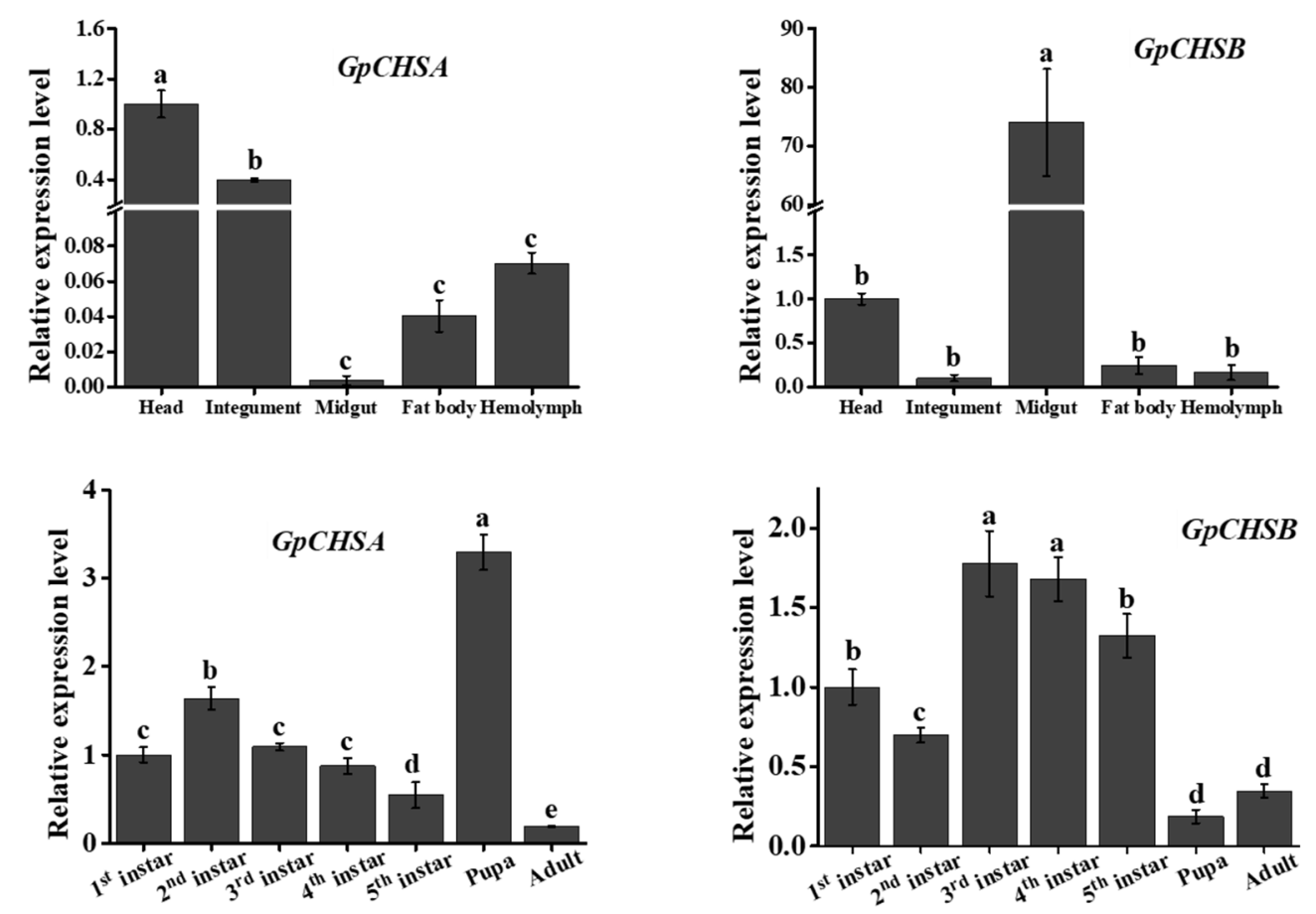

Figure 4. Analysis of GpCHSA and GpCHSB expression in different developmental stages and tissues of fifth instar larvae using RT-qPCR. GpRpl32 was used to normalize the data that are showed as mean \pm standard error, the mean is the triple independent repeats. The $2^{-\Delta \Delta C t}$ method was adopted to calculate the relative expression level. Differences among triple repeats were analyzed using one-way analysis of variance (Systat, Inc., Evanston, IL) with Tukey's post-hoc test using R version 4.0.0. Different letters $(\mathrm{a}, \mathrm{b}, \mathrm{c}, \mathrm{d}, \mathrm{e})$ represent significant difference $(p<0.05)$.

\subsection{Inhibition of GpCHSA Expression Could Downregulate Expression of Its Downstream Genes}

To further explore the function of GpCHSA in the integument chitin synthesis pathway, dsRNAs were injected into larvae at the first day of fifth instar stage. The expression level of GpCHSA was measured at 24, 48, and $72 \mathrm{~h}$ after injection with dsCHSA using RT-qPCR. Results revealed that the expression of GpCHSA was significantly downregulated at $48 \mathrm{~h}$ after injection as compared with the control group (Figure 5). Moreover, the expression of GpCDA1, GpCDA2, and GpCHT3a showed similarly affected expression patterns with GpCHSA after knockdown of GpCHSA. Briefly, expression of GpCHSA was significantly upregulated at $24 \mathrm{~h}$ and downregulated at $48 \mathrm{~h}$ after injection with dsRNA (Figure 5), indicating GpCHSA plays a vital role in regulating GpCDA1, GpCDA2, and GpCHT3a expression. 

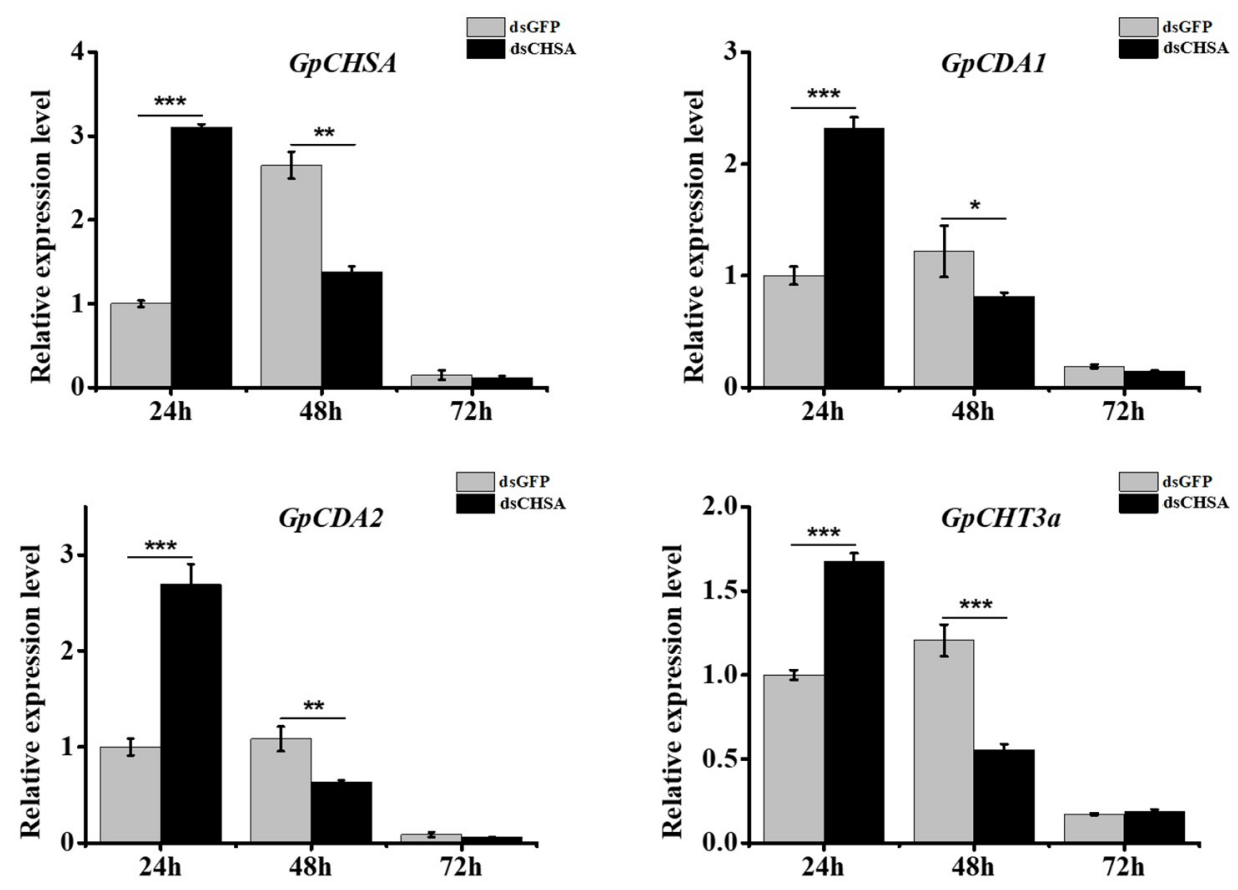

Figure 5. The analysis of expression patterns of GpCHSA and its downstream genes post-injection with dsCHSA in the integument at different times using RT-qPCR. GpRpl32 was used to normalize the data that are showed as mean \pm standard error, the mean is the triple independent repeats. The $2^{-\Delta \Delta C t}$ method was adopted to calculate the relative expression level. Differences among triple repeats were analyzed using one-way analysis of variance (Systat, Inc., Evanston, IL) with Tukey's post-hoc test using $\mathrm{R}$ version 4.0.0. Asterisks represent the significant difference, as follows: ${ }^{*} p<0.05 ;{ }^{* *} p<0.01$; *** $p<0.001$.

\subsection{RNAi of GpCHSB Resulted in Significant Downregulation of Its Downstream Genes}

The method used to assess RNAi effect of GpCHSB was the same as was used to assess effects of GpCHSA knockdown described above. Expression level of GpCHSB was measured at 24, 48, and $72 \mathrm{~h}$ after injection with dsCHSB using RT-qPCR. Results revealed that GpCHSB expression was significantly downregulated at $48 \mathrm{~h}$ after injection with dsRNA (Figure 6). Moreover, the expression levels of GpCDA5, GpCHT3b, and GpCHT-h were significantly downregulated at $48 \mathrm{~h}$ after knockdown of GpCHSB (Figure 6), while GpCDA5 and GpCHT- $h$ were upregulated at $72 \mathrm{~h}$ post-injection.

\subsection{RNAi of GpCHSA and GpCHSB Resulted in an Adverse Effect on G. pyloalis Development}

To further confirm the functions of GpCHSA and GpCHSB, adult and pupal phenotypes of $G$. pyloalis were assessed after injection with dsCHSA and dsCHSB. Results showed that knockdown of GpCHSA and GpCHSB adversely affected G. pyloalis wing development, including abnormal wing stretching and partial loss (Figure 7). However, pupation of fifth instar larvae was not affected by dsRNA injection (Figure 7). 

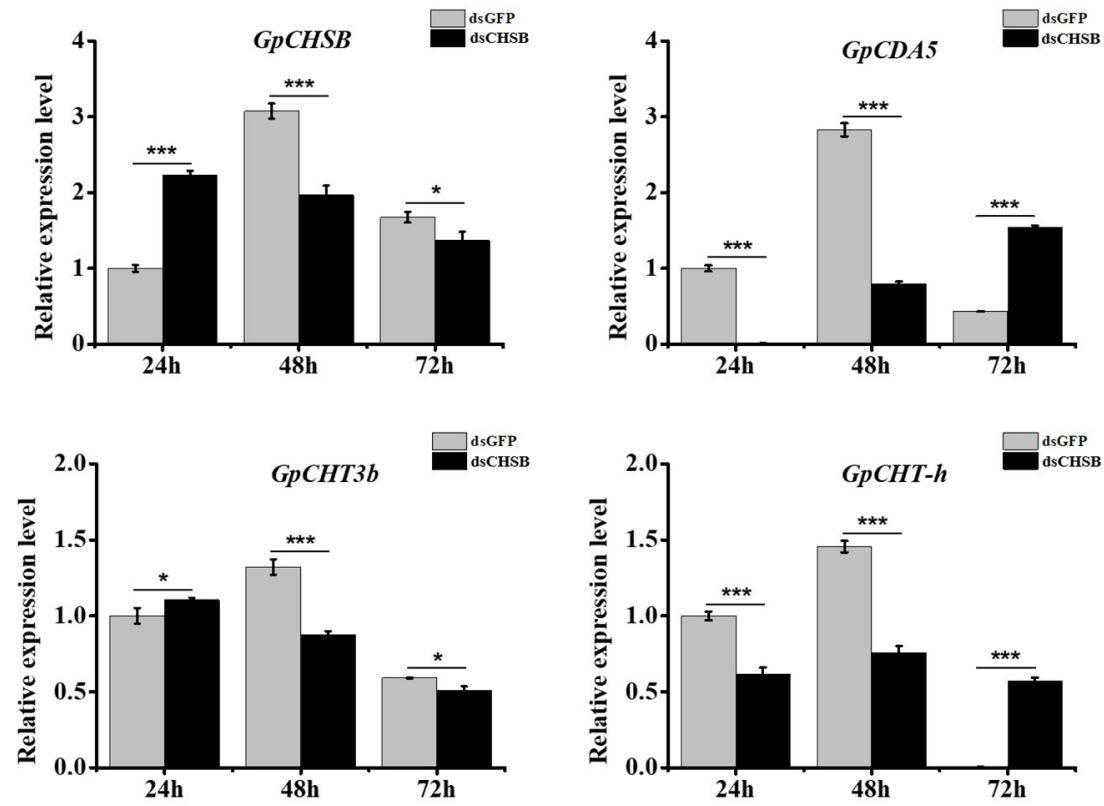

Figure 6. The analysis of expression patterns of GpCHSB and its downstream genes after injection with dsCHSB in the midgut at different times using RT-qPCR. GpRpl32 was used to normalize the data that are showed as mean \pm standard error, the mean is the triple independent repeats. The $2^{-\Delta \Delta C t}$ method was adopted to calculate the relative expression level. Differences among triple repeats were analyzed using one-way analysis of variance (Systat, Inc., Evanston, IL, USA) with Tukey's post-hoc test using R version 4.0.0. Asterisks represent the significant difference, as follows: ${ }^{*} p<0.05 ;{ }^{* * *} p<0.001$.

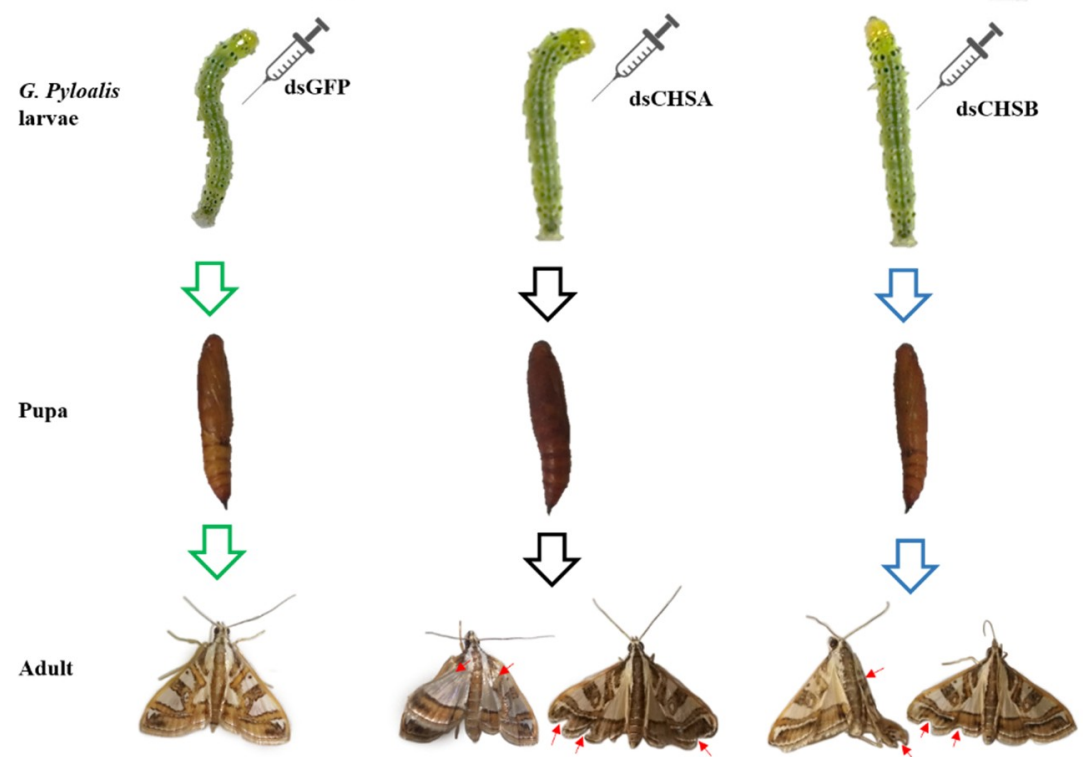

Figure 7. Representative phenotypes of G. pyloalis after dsRNA treatment. The phenotypes are taken after metamorphosis using Canon, PowerShot SX720.

\subsection{DFB Treatment Could Affect Expression of GpCHSA, GpCHSB, and Their Downstream Genes}

In order to further validate findings observed after injection with dsCHSA and dsCHSB, fourth instar larvae of G. pyloalis after treatment with DFB was assessed at different times. Results revealed that GpCHSA expression was significantly upregulated at 24 and $48 \mathrm{~h}$ post-exposure to DFB in the integument (Figure 8). Moreover, relative expression levels of GpCDA1, GpCDA2 and GpCHT3a 
involved in the integument chitin metabolism pathway were also significantly upregulated at $24 \mathrm{~h}$ post-treatment with DFB (Figure 8).

The relative expression level of $G p C H S B$ was significantly down-regulated at $12 \mathrm{~h}$ post-exposure to DFB in the midgut. Subsequently, expression was upregulated at 24 and $48 \mathrm{~h}$ post-exposure with DFB did not significant differ (Figure 8). Moreover, expression levels of GpCDA5 and GpCHT- $h$ were significantly upregulated at $12 \mathrm{~h}$ post-exposure to $\mathrm{DFB}$, while $\mathrm{G} p \mathrm{CHT} 3 \mathrm{~b}$ was downregulated (Figure 8). After $12 \mathrm{~h}$ of DFB exposure, three downstream genes of G $p C H S B$ were downregulated. However, the same downstream genes were upregulated at $48 \mathrm{~h}$ post-DFB treatment (Figure 8 ).

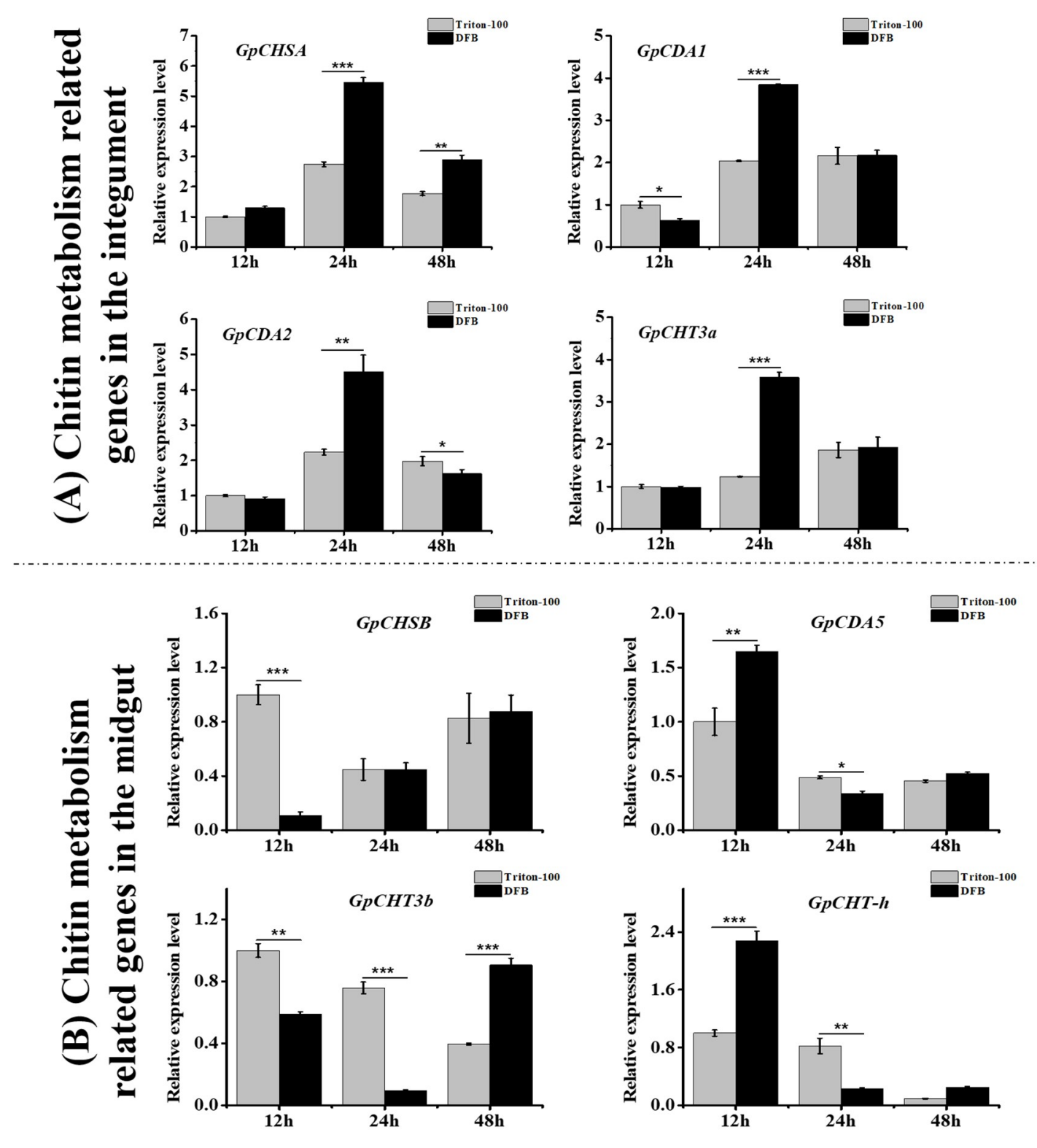

Figure 8. Assessment of expression levels of GpCHSA, GpCHSB, and their downstream genes post-DFB treatment. (A) Expression of GpCHSA and its downstream genes in the integument. (B) Expression of GpCHSB and its downstream genes in the midgut. GpRpl32 was used to normalize the data that are showed as mean \pm standard error, the mean is the triple independent repeats. The $2^{-\Delta \Delta C t}$ method was adopted to calculate the relative expression level. Differences among triple repeats were analyzed using one-way analysis of variance (Systat, Inc., Evanston, IL) with Tukey's post-hoc test using R version 4.0.0. Asterisks represent the significant difference, as follows: ${ }^{*} p<0.05 ;{ }^{* *} p<0.01 ;{ }^{* * *} p<0.001$. 


\subsection{DFB Treatment Adversely Affected G. pyloalis Development}

In order to assess effects of DFB treatment on G. pyloalis development and mortality, a leaf-dip bioassay was conducted. Results indicated abnormal molting occurred after treatment with DFB (Figure 9A,B). Moreover, cumulative mortality of G. pyloalis larvae was significantly increased at $48 \mathrm{~h}$ $(62 \%)$ and $72 \mathrm{~h}(90 \%)$ after exposure to DFB as compared with control insects (Figure $9 \mathrm{C}$ ).

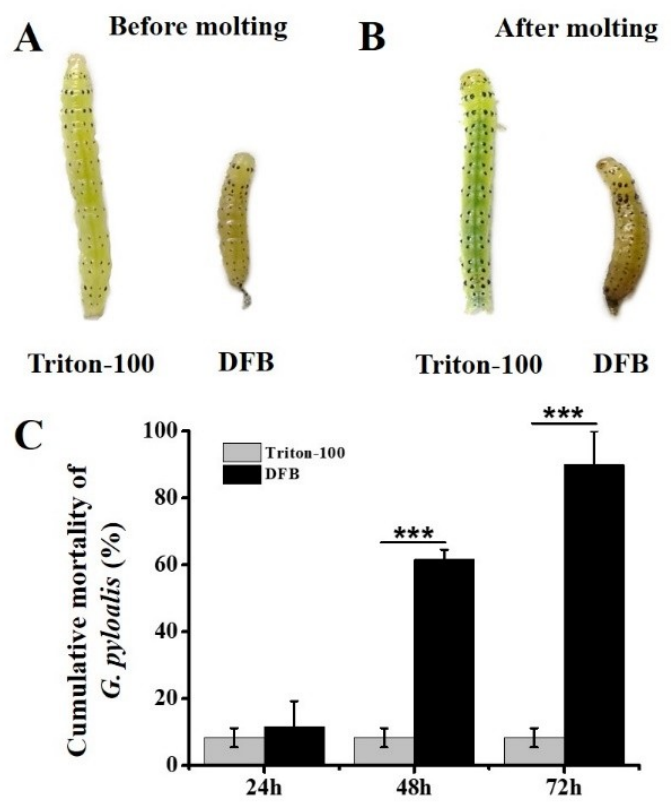

Figure 9. The effect of DFB on G. pyloalis development. (A-B) G. pyloalis phenotypes post-DFB and Triton-100 (control) treatment before and after molting. (C) Cumulative mortality of G. pyloalis after exposure to DFB and Triton-100. Differences among triple repeats were analyzed using one-way analysis of variance (Systat, Inc., Evanston, IL) with Tukey's post-hoc test using R version 4.0.0. Asterisks represent the significant difference, as follows: ${ }^{* *} p<0.001$.

\section{Discussion}

As use of pesticides has become widespread, G. pyloalis insecticide resistance has become severity in recent years. Hence, it is necessary to find alternate effective and environmentally friendly methods to solve the problem. Chitin is an essential component of the cuticle and PM of G. pyloalis, which serves as an initial barrier that protects G. pyloalis from pesticides. To identify the genes involved in chitin biosynthetic pathway, the integument, midgut, and whole larvae of G. pyloalis were sequenced using RNA-Seq method in our previous study [28]. A total of 19 genes were identified that encode chitin metabolism-related enzymes, and GpCHSA and GpCHSB related to the chitin biosynthesis are two of them. In this study, to further validate the function of GpCHSA and GpCHSB in the chitin metabolism pathway, RNAi and the inhibitor of chitin synthesis DFB were used.

It was reported that many enzymes were involved in the chitin metabolism progress. Chitin synthase (CHS) plays a key role in chitin biosynthesis during insect growth and metamorphosis in Diptera, Coleoptera, Lepidoptera, Hemiptera, and Hymenoptera [8]. In fungal species, there are more than 20 fungal CHS genes have been identified, while only two CHS genes (CHSA and CHSB) occur in insects [4]. CHSA is highly expressed in the cuticle and is essential for the formation of integument. CHSB is greatly expressed in the midgut and is an important component of the PM [30]. However, CHS genes of G. pyloali have not been well studied. In this study, complete coding sequences of GpCHSA and GpCHSB were identified from our previously constructed G. pyloali transcriptome database. GpCHSA and GpCHSB contain two conserved catalytic domains, EDR and QRRRW, which are involved in chitin 
synthesis and conserved among different species. This indicated their likely function in G. pyloali chitin synthesis (Figure 1).

GpCHSA and GpCHSB were divided into two different groups via phylogenetic analysis, indicating that the two genes are likely involved in two different chitin metabolism pathways. This finding was further validated by the observation that expression levels of GpCHSA were higher in the integument as compared with others, while higher expression of GpCHSB in the midgut (Figures 3 and 4). These results are consistent with previous studies involving other species [31-34]. Moreover, significant differences between expression levels of GpCHSA and GpCHSB were observed throughout all selected development stages, which indicated that the two $\mathrm{CHS}$ genes likely played important roles in the whole life of G. pyloali (Figure 4). Moreover, enhanced expression of GpCHSA in the pupa and relatively high expression levels of $G p C H S B$ throughout larval stages validated their roles in chitin metabolism pathways of the integument and midgut, respectively (Figure 4).

RNAi has been proved to be an efficient molecular biology technique, and has been widely used to study gene function in insects. RNAi also has been considered as a potential mean to control pests [35]. Since the chitin synthesis pathway does not exist in vertebrates and plants, RNAi targeting of chitin synthases is an attractive potential means to control insect populations [36]. It has been widely reported that knockdown of CHS genes using dsRNA is feasible in lepidopteran insects. For example, feeding of Helicoverpa armigera larvae with $\mathrm{HaCHSA}$ dsRNA resulted in reduced body weight, growth and pupation rates [37]. Knockdown of SeCHSA in Spodoptera exigua larvae using dsRNA reduced survival rates in larvae, prepupa and pupa [38]. In this study, larvae were injected with dsRNA on the first day of fifth instar stage to study the function of GpCHSA and GpCHSB in the chitin metabolic pathway. Significant downregulation of GpCHSA and GpCHSB at $48 \mathrm{~h}$ post-injection indicated that use of dsRNA was effective (Figures 5 and 6 ), and the significant decrease of the expression of downstream genes of GpCHSA and GpCHSB, including GpCDA1, GpCDA2, GpCHT3a and GpCDA5, GpCHT-h, GpCHT3b, respectively, indicated $G p C H S A$ and $G p C H S B$ played vital roles in the regulation of their expression. Moreover, we found that G. pyloali wing development was affected by dsCHSA and dsCHSB (Figure 7), which was consistent with the results of other species that also occurred abnormal development after knockdown of CHS expression [17,39].

DFB as a chitin synthesis inhibitor has been recommended for spraying forage crops in order to protect against several pests [21,40]. DFB is effective because it causes abnormal procuticle deposition and abortive molting in pests [12,23,41]. Its function mainly reduces chitin content in insects, and frequently leads to the upregulation of CHSA. This may be caused by a feedback regulatory mechanism that attempts to compensate for decreased enzyme content [12,30]. In this study, GpCHSA was significantly upregulated approximately 2 fold at $24 \mathrm{~h}$ after exposure to DFB (Figure 8), which was consistent with previous reports [12,23]. However, levels of GpCHSB were not significantly altered (Figure 8), which reflected that GpCHSA might play a more important role in the response to DFB treatment than GpCHSB. It has been shown previously that DFB affects species differently. For example, no effects of DFB were observed in D. melanogaster [42] and T. castaneum [43]. Moreover, downstream genes of $G p C H S A$, including GpCDA1, GpCDA2 and GpCHT3a, increased post-upregulation of GpCHSA expression (Figure 8). This further validated the results of RNAi, and indicated that GpCHSA was involved in regulating expression of its downstream genes. Though the expression of GpCHSB was not significantly altered by DFB treatment, but the same trend of the downstream genes of GpCHSB with itself at 24 and $48 \mathrm{~h}$ after DFB treatment (Figure 8), including GpCDA5, GpCHT- $h$, and GpCHT3b, indicated their close relationship with G $p C H S B$. Furthermore, larvae fed DFB displayed abnormal molting and increased mortality as compared with control insects (Figure 9), indicating that DFB treatment affected G. pyloali development by disturbing the chitin metabolism pathway. These results further suggest the role of GpCHSA and GpCHSB in the chitin metabolism pathway.

Based on results described above, it was reasonable to conclude that GpCHSA and GpCHSB were involved in G. pyloali cuticle and PM chitin metabolism, respectively. GpCHSA regulated expression of its downstream genes $G p C D A 1, G p C D A 2$, and GpCHT3a. GpCDA1 and GpCDA2 participates in cuticle 
chitin deacetylation, and GpCHT3a in cuticle chitin degradation (Figure 10). Moreover, GpCHSB could regulate the expression of its downstream genes GpCDA5, GpCHT- $h$ and GpCHT3b. GpCDA5 regulated PM chitin deacetylation, and GpCHT- $h$ and GpCHT3b affected chitin degradation in the PM (Figure 10). Furthermore, GpCHSA and GpCHSB played a critical role molting and wing development in G. pyloalis via regulation of chitin metabolism. Taken together, these findings indicate that GpCHSA and GpCHSB may be attractive new targets for G. pyloalis control.

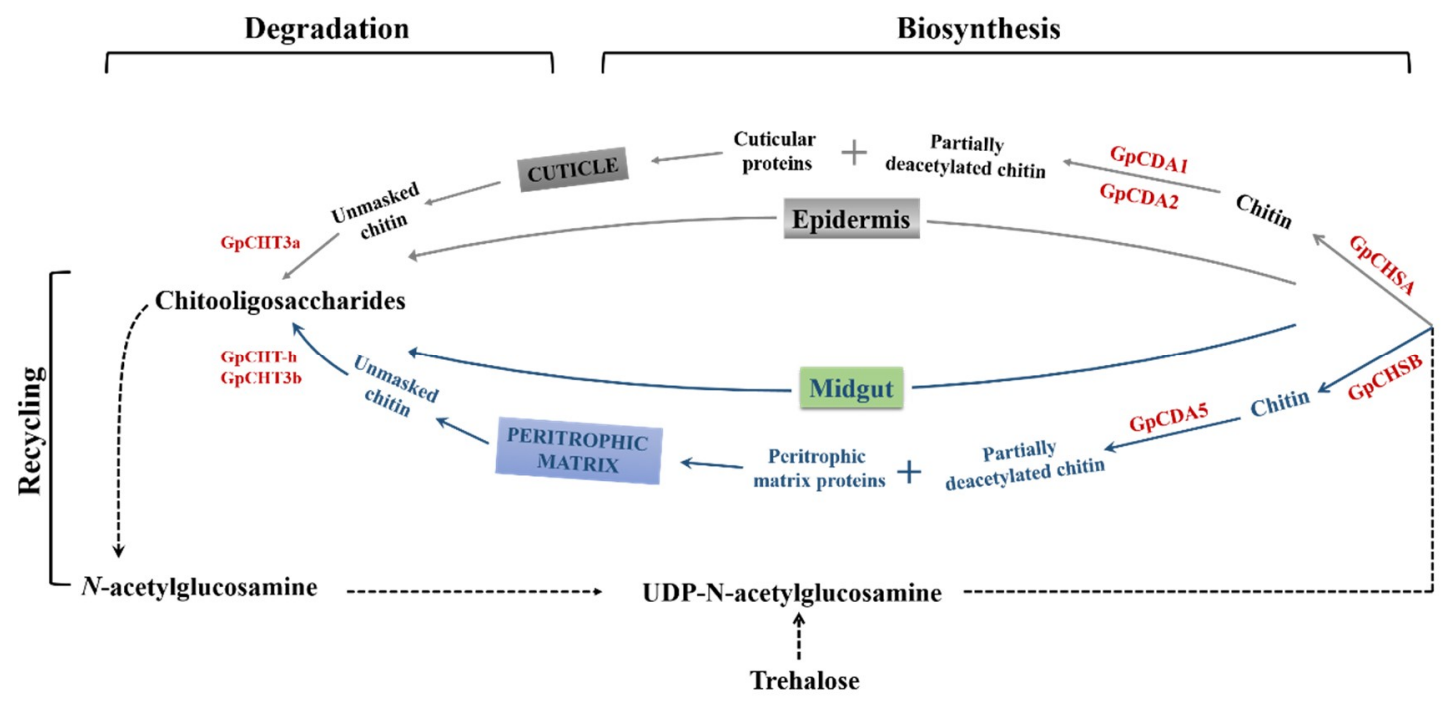

Figure 10. The hypothesis of the role of GpCHSA and GpCHSB and their downstream genes in chitin metabolism in G. pyloalis. GpCHSA and GpCHSB are involved in cuticle and PM chitin metabolism, respectively. GpCHSA regulates the expression of GpCDA1, GpCDA2 and GpCHT3a. GpCHSB regulates expression of GpCDA5, GpCHT-h and GpCHT3b. Genes analyzed in this study were highlighted with the red color.

\section{Materials and Methods}

\subsection{Bioinformatics Analysis}

ORF finder (https://www.ncbi.nlm.nih.gov/orfnder/) was used to predicte the open reading frames (ORFs) of putative GpCHSA and GpCHSB genes. ExPASy (https://web.expasy.org/compute_pi/) was used to predicte the theoretical isoelectric point (pI) and molecular weight of each enzyme. DNAMAN 8.0 software (Lynnon Corporation, Quebec, Canada) was used to perform the multiple alignments of various protein sequences. Conserved motifs were predicted by using SMART software (http://smart.embl-heidelberg.de/). Phylogenetic analysis was conducted using MEGA-X software and the neighbor-joining method with 1,000 bootstrap replications. The protein sequences of GpCHS homologs of 19 other species were acquired from GenBank (http://www.ncbi.nlm.nih.gov/). GenBank IDs of each protein are listed in Table S1.

\subsection{Insect Rearing and Sample Preparation}

G. pyloalis were maintained in the Key Laboratory of Silkworm and Mulberry Genetic Improvement, Ministry of Agriculture, Sericultural Research Institute, Chinese Academy of Agricultural Science, Zhenjiang, Jiangsu, China. The larvae were reared in an insect-rearing room with fresh mulberry leaves. The rear conditions are $25 \pm 1{ }^{\circ} \mathrm{C}, 60-80 \%$ relative humidity, and a $14 \mathrm{~h}$ light and $10 \mathrm{~h}$ dark photoperiod.

The different development stages of G. pyloalis has been classified based on insect morphological features. Ten samples were mixed to minimize individual genetic differences in detecting the relative expression level in different life stages. Different tissues of 50 larvae in fifth instar were dissected for 
detecting relative expression levels in different tissues. All samples were kept in $-80{ }^{\circ} \mathrm{C}$ for further use. Triple replicates of each gene in these samples were performed.

\subsection{Total RNA Extraction and cDNA Synthesis}

TRIzol reagent (Invitrogen, New York, NY, USA) was used to extract the total RNA in accordance with the manufacturer's protocols. The NanoDrop 2000 spectrophotometer (Thermo Fisher Scientific, New York, NY, USA) was used to perform the RNA quantification. RNA purity was determined by assessing optical density (OD) absorbance ratios at $\mathrm{OD}_{260 / 280}$ and $\mathrm{OD}_{260 / 230}$. The integrity of RNA was analyzed via $1 \%$ agarose gel electrophoresis with ethidium bromide staining. Reverse transcription was performed using a PrimeScript ${ }^{\mathrm{TM}} \mathrm{RT}$ reagent Kit with a gDNA Eraser (TaKaRa Biotechnology Co. Ltd., Dalian, China). Briefly, reaction was incubated at $37^{\circ} \mathrm{C}$ for $15 \mathrm{~min}$ and then $85^{\circ} \mathrm{C}$ for $5 \mathrm{~s}$. Synthesized cDNA was preserved at $-20{ }^{\circ} \mathrm{C}$ for further use.

\subsection{Quantitative Reverse Transcription PCR (RT-qPCR)}

NCBI Primer-BLAST software (https://www.ncbi.nlm.nih.gov/tools/primer-blast/) was used to design the specific primers that were used for RT-qPCR analysis and shown in Table S2. The reaction was analyzed using a QuantStudio ${ }^{\mathrm{TM}}$ Real-Time PCR system (Thermo Fisher Scientific, Applied Biosystems, New York, NY, USA). Each reaction used $15 \mu \mathrm{L}$ reaction system containing $1.5 \mu \mathrm{L}$ cDNA, $7.5 \mu \mathrm{L}$ TB Green Fast qPCR Mix (TaKaRa Biotechnology Co. Ltd., Dalian, China), $0.6 \mu \mathrm{L}$ of each gene-specific primer $(0.4 \mu \mathrm{M}), 0.3 \mathrm{uL}$ of ROX Reference Dye II and $4.5 \mu \mathrm{L}$ of RNase free $\mathrm{H}_{2} \mathrm{O}$. G. pyloali ribosomal protein $L 32$ (GpRpl32) was used as reference standard gene [44]. The $2^{-\Delta \Delta C t}$ method was used to calculate the relative expression level of each gene. Statistical analysis was conducted using $\mathrm{R}$ version 4.0.0. The triplicate data of different groups were analyzed via One-way ANOVA with Tukey's posttest.

\section{5. dsRNA Synthesis and Injection}

To ensure the RNAi efficiency, two specific targets of the functional domain of GpCHSA and GpCHSB genes were designed. The specific target of green fluorescent protein (GFP) was selected as negative treatment. Primers used to synthesize dsRNA by Sangon Biotechnology (Shanghai, China) are listed in Table S3. The in vitro Transcription T7 Kit (for dsRNA synthesis; TaKaRa Biotechnology Co. Ltd., Dalian, China) was used to synthesize dsCHSA, dsCHSB, and dsGFP according to the manufacturer's instructions. The integrity of the dsRNA was determined by $3 \%$ agarose gel electrophoresis. The quality and concentration of purified dsRNA were measured using a NanoDrop 2000 spectrophotometer (Thermo Fisher Scientific, NY, USA). Newly synthesized dsRNA was saved in $-80^{\circ} \mathrm{C}$ for further use.

$\mathrm{dsCHSA}$ and dsCHSB were dissolved in DEPC water to a final concentration of $2.0 \mu \mathrm{g} / \mu \mathrm{L}$. Equal volumes of the two targets were mixed, and $1.0 \mu \mathrm{L}$ of the mixture dsRNAs were injected into each larva on the first day of fifth instar development period using microneedles. Thirty (30) larvae were under dsRNA and $\mathrm{ddH}_{2} \mathrm{O}$ treatment in each group. Expression levels of the two genes and their downstream genes in the integument and midgut were analyzed using RT-qPCR after RNAi at 24, 48 , and $72 \mathrm{~h}$ post-injection, respectively. Triple biological replicates were done for each group. The method used to investigate phenotypes of G. pyloalis in different stages after RNAi was the same with sample preparation as descripted above.

\subsection{Leaf Dip Bioassay}

To determine the effect of DFB on GpCHSA and GpCHSB and their downstream genes expression, the mortality rates of fourth instar larvae of G. pyloalis were determined using a leaf-dip bioassay using a previous protocol with some modifications [23]. Briefly, mulberry leaves $9-\mathrm{cm}$ in diameter were dipped in the final concentration $500 \mu \mathrm{g} / \mathrm{mL}$ of DFB (LKT Laboratories Inc., Saint Paul, MN, USA) that was dissolved in $0.1 \%$ Triton- 100 for 3 min and air dried. Leaves treated with $0.1 \%$ Triton- 100 
were used as controls. Thirty of fourth instar larvae exposed to DFB and Triton-100 were kept in the insect-rearing room for 24,48 , and $72 \mathrm{~h}$ to investigate phenotypic effects of treatment and cumulative mortality, respectively. Surviving larvae were collected for further analysis. The experiment was performed using triple biological replicates.

Supplementary Materials: The following are available online at http://www.mdpi.com/1422-0067/21/13/4656/s1.

Author Contributions: Conceived and designed the experiments: Z.-M.S., F.-A.W. Performed the experiments: Z.-M.S., Y.-J.L., J.-H.D., Z.-X.L. and X.-R.Z. Analyzed the data: Z.-M.S. Contributed reagents/materials/analysis tools: F.-A.W., J.W. and S.S. Wrote the paper: Z.-M.S. All authors have read and agreed to the published version of the manuscript.

Funding: This research was funded by the Special Fund for China Agriculture Research System (CARS-18), the Postgraduate Research \& Practice Innovation Program of Jiangsu Province (KYCX20_2935), and the National Natural Science Foundation of China (31500312).

Conflicts of Interest: The authors declare no conflict of interest.

\section{References}

1. Watanabe, H.; Kurihara, Y.; Wang, Y.X.; Shimizu, T. Mulberry pyralid, Glyphodes pyloalis: Habitual host of nonoccluded viruses pathogenic to the silkworm, Bombyx mori. J. Invertebr. Pathol. 1988, 52, 401-408. [CrossRef]

2. Yazdani, E.; Sendi, J.J;; Aliakbar, A.; Senthil-Nathan, S. Effect of Lavandula angustifolia essential oil against lesser mulberry pyralid Glyphodes pyloalis Walker (Lep: Pyralidae) and identification of its major derivatives. Pestic. Biochem. Physiol. 2013, 107, 250-257. [CrossRef]

3. Merzendorfer, H.; Zimoch, L. Chitin metabolism in insects: Structure, function and regulation of chitin synthases and chitinases. J. Exp. Biol. 2003, 206, 4393-4412. [CrossRef] [PubMed]

4. Merzendorfer, $\mathrm{H}$. The cellular basis of chitin synthesis in fungi and insects: Common principles and differences. Eur. J. Cell. Biol. 2011, 90, 759-769. [CrossRef] [PubMed]

5. Rahman, M.A.; Halfar, J. First evidence of chitin in calcified coralline algae: New insights into the calcification process of Clathromorphum compactum. Sci. Rep. 2014, 4, 6162. [CrossRef] [PubMed]

6. Rahman, M.A.; Halfar, J.; Adey, W.H.; Nash, M.; Paulo, C.; Dittrich, M. The role of chitin-rich skeletal organic matrix on the crystallization of calcium carbonate in the crustose coralline alga Leptophytum foecundum. Sci. Rep. 2019, 9, 11869. [CrossRef]

7. Mansur, J.F.; Alvarenga, E.S.L.; Mansur, J.F.; Franco, T.A.; Ramos, I.B.; Masuda, H.; Melo, A.C.A.; Moreira, M.F. Effects of chitin synthase double-stranded RNA on molting and oogenesis in the Chagas disease vector Rhodnius prolixus. Insect Biochem. Mol. Biol. 2014, 51, 110-121. [CrossRef]

8. Zhu, K.Y.; Merzendorfer, H.; Zhang, W.Q.; Zhang, J.Z.; Muthukrishnan, S. Biosynthesis, turnover, and functions of chitin in insects. Annu. Rev. Entomol. 2016, 61, 177-196. [CrossRef]

9. Bulawa, C.E. Genetics and molecular biology of chitin synthesis in fungi. Annu. Rev. Microbiol. 1993, 47, 505-534. [CrossRef]

10. Silverman, S.J.; Sburlati, A.; Slater, M.L.; Cabib, E. Chitin synthase 2 is essential for septum formation and cell division in Saccharomyces cerevisiae. Proc. Natl. Acad. Sci. USA 1988, 85, 4735-4739. [CrossRef]

11. Dorfmueller, H.C.; Ferenbach, A.T.; Borodkin, V.S.; Aalten, D.M.F. A structural and biochemical model of processive chitin synthesis. J. Biol. Chem. 2014, 289, 23020-23028. [CrossRef] [PubMed]

12. Zhang, J.z.; Zhu, K.Y. Characterization of a chitin synthase cDNA and its increased mRNA level associated with decreased chitin synthesis in Anopheles quadrimaculatus exposed to diflubenzuron. Insect Biochem. Mol. Biol. 2006, 36, 712-725. [CrossRef] [PubMed]

13. Tellam, R.L.; Vuocolo, T.; Johnson, S.E.; Jarmey, J.; Pearson, R.D. Insect chitin synthase: cDNA sequence, gene organization and expression. Eur. J. Biochem. 2000, 267, 6025-6043. [CrossRef] [PubMed]

14. Gagou, M.E.; Kapsetaki, M.; Turberg, A.; Kafetzopoulos, D. Stage-specific expression of the chitin synthase DmeChSA and DmeChSB genes during the onset of Drosophila metamorphosis. Insect. Biochem. Mol. Biol. 2002, 32, 141-146. [CrossRef]

15. Ampasala, D.R.; Zheng, S.C.; Zhang, D.Y.; Ladd, T.; Doucet, D.; Krell, P.J.; Retnakaran, A.; Feng, Q.L. An epidermis-specific chitin synthase CDNA in Choristoneura fumiferana: Cloning, characterization, developmental and hormonal-regulated expression. Arch. Insect Biochem. Physiol. 2011, 76, 83-96. [CrossRef] 
16. Zhang, X.; Zhang, J.Z.; Park, Y.; Zhu, K.Y. Identification and characterization of two chitin synthase genes in African malaria mosquito, Anopheles gambiae. Insect Biochem. Mol. Biol. 2012, 42, 674-682. [CrossRef]

17. Zhang, J.Z.; Liu, X.J.; Zhang, J.Q.; Li, A.Q.; Sun, Y.; Guo, Y.P.; Ma, E.B.; Zhu, K.Y. Silencing of two alternative splicing-derived mRNA variants of chitin synthase 1 gene by RNAi is lethal to the oriental migratory locust, Locusta migratoria manilensis (Meyen). Insect Biochem. Mol. Biol. 2010, 40, 824-833. [CrossRef]

18. Arakane, Y.; Muthukrishnan, S.; Kramer, K.J.; Specht, C.A.; Tomoyasu, Y.; Lorenzen, M.D.; Kanost, M.; Beeman, R.W. The Tribolium chitin synthase genes TcCHS1 and TcCHS2 are specialized for synthesis of epidermal cuticle and midgut peritrophic matrix. Insect Mol. Biol. 2005, 14, 453-463. [CrossRef]

19. Ashfaq, M.; Sonoda, S.; Tsumuki, H. Developmental and tissue-specific expression of CHS1 from Plutella xylostella and its response to chlorfluazuron. Pestic. Biochem. Physiol. 2007, 89, 20-30. [CrossRef]

20. Zimoch, L.; Merzendorfer, H. Immunolocalization of chitin synthase in the tobacco hornworm. Cell Tissue Res. 2002, 308, 287-297. [CrossRef]

21. Cohen, E. Chitin synthesis and inhibition: A revisit. Pest. Manag. Sci. 2001, 57, 946-950. [CrossRef] [PubMed]

22. Merzendorfer, H. Insect chitin synthases: A review. J. Comp. Physiol. B 2005, 176, 1-15. [CrossRef] [PubMed]

23. Xia, W.K.; Ding, T.B.; Niu, J.Z.; Liao, C.Y.; Zhong, R.; Yang, W.J.; Liu, B.; Dou, W.; Wang, J.J. Exposure to diflubenzuron results in an up-regulation of a chitin synthase 1 gene in citrus red mite, Panonychus citri (Acari: Tetranychidae). Int. J. Mol. Sci. 2014, 15, 3711-3728. [CrossRef] [PubMed]

24. Ye, C.; Jiang, Y.D.; An, X.; Yang, L.; Shang, F.; Niu, J.Z.; Wang, J.J. Effects of RNAi-based silencing of chitin synthase gene on moulting and fecundity in pea aphids (Acyrthosiphon pisum). Sci. Rep. 2019, 9, 3694. [CrossRef]

25. Zhang, X.; Zhang, J.; Zhu, K.Y. Chitosan/double-stranded RNA nanoparticle-mediated RNA interference to silence chitin synthase genes through larval feeding in the African malaria mosquito (Anopheles gambiae). Insect Mol. Biol. 2010, 19, 683-693. [CrossRef]

26. Lu, Z.J.; Huang, Y.L.; Yu, H.Z.; Li, N.Y.; Xie, Y.X.; Zhang, Q.; Zeng, X.D.; Hu, H.; Huang, A.J.; Yi, L.; et al. Silencing of the chitin Synthase gene is lethal to the asian citrus Psyllid, Diaphorina citri. Int. J. Mol. Sci. 2019, 20, 3734. [CrossRef]

27. Shang, F.; Xiong, Y.; Xia, W.K.; Wei, D.D.; Wei, D.; Wang, J.J. Identification, characterization and functional analysis of a chitin synthase gene in the brown citrus aphid, Toxoptera citricida (Hemiptera, Aphididae). Insect Mol. Biol. 2016, 25, 422-430. [CrossRef]

28. Shao, Z.M.; Li, Y.J.C.; Zhang, X.R.; Chu, J.; Ma, J.H.; Liu, Z.X.; Wang, J.; Sheng, S.; Wu, F.A. Identification and functional study of chitin metabolism and detoxification-related genes in Glyphodes pyloalis Walker (Lepidoptera: Pyralidae) based on transcriptome analysis. Int. J. Mol. Sci. 2020, 21, 1094. [CrossRef]

29. Kamst, E.; Spaink, H. Functional domains in the chitin oligosaccharide synthase NodC and related beta-polysaccharide synthases. Trends Glycosci. Glyc. 1999, 11, 187-199. [CrossRef]

30. Bansal, R.; Mian, M.A.R.; Mittapalli, O.; Michel, A.P. Characterization of a chitin synthase encoding gene and effect of diflubenzuron in soybean aphid, Aphis glycines. Int. J. Biol. Sci. 2012, 8, 1323-1334. [CrossRef]

31. Liu, X.J.; Zhang, H.H.; Li, S.; Zhu, K.Y.; Ma, E.B.; Zhang, J.Z. Characterization of a midgut-specific chitin synthase gene (LmCHS2) responsible for biosynthesis of chitin of peritrophic matrix in Locusta migratoria. Insect Biochem. Mol. Biol. 2012, 42, 902-910. [CrossRef] [PubMed]

32. Zhou, W.W.; Fang, Y.; Kong, L.F.; Li, X.; Sima, Y.H.; Xu, S.Q. Chitin synthase a: A novel epidermal development regulation gene in the larvae of Bombyx mori. Mol. Biol. Rep. 2014, 41, 4177-4186. [CrossRef] [PubMed]

33. Qu, M.; Yang, Q. A novel alternative splicing site of class A chitin synthase from the insect Ostrinia furnacalis-Gene organization, expression pattern and physiological significance. Insect Biochem. Mol. Biol. 2011, 41, 923-931. [CrossRef] [PubMed]

34. Chen, X.; Tian, H.; Zou, L.; Tang, B.; Hu, J.; Zhang, W. Disruption of Spodoptera exigua larval development by silencing chitin synthase gene A with RNA interference. Bull. Entomol. Res. 2008, 98, 613-619. [CrossRef]

35. Huvenne, H.; Smagghe, G. Mechanisms of dsRNA uptake in insects and potential of RNAi for pest control: A review. J. Insect Physiol. 2010, 56, 227-235. [CrossRef] [PubMed]

36. Merzendorfer, H. Chitin synthesis inhibitors: Old molecules and new developments. Insect Sci. 2013, 20, 121-138. [CrossRef] 
37. Jin, S.X.; Singh, N.D.; Li, L.B.; Zhang, X.L.; Daniell, H. Engineered chloroplast dsRNA silences cytochrome p450 monooxygenase, V-ATPase and chitin synthase genes in the insect gut and disrupts Helicoverpa armigera larval development and pupation. Plant. Biotechnol. J. 2015, 13, 435-446. [CrossRef]

38. Tian, H.G.; Peng, H.; Yao, Q.; Chen, H.G.; Xie, Q.; Tang, B.; Zhang, W.Q. Developmental control of a Lepidopteran pest Spodoptera exigua by ingestion of bacteria expressing dsRNA of a non-midgut gene. PLOS ONE 2009, 4, e6225. [CrossRef]

39. Yang, X.S.; Yin, Q.; Xu, Y.; Li, X.X.; Sun, Y.; Ma, L.; Zhou, D.; Shen, B. Molecular and physiological characterization of the chitin synthase $B$ gene isolated from Culex pipiens pallens (Diptera: Culicidae). Parasites Vectors 2019, 12, 614. [CrossRef]

40. Abo-Elghar, G.E.; Fujiyoshi, P.; Matsumura, F. Significance of the sulfonylurea receptor (SUR) as the target of diflubenzuron in chitin synthesis inhibition in Drosophila melanogaster and Blattella germanica. Insect Biochem. Mol. Biol. 2004, 34, 743-752. [CrossRef]

41. Mulder, R.; Gijswijt, M.J. The laboratory evaluation of two promising new insecticides which interfere with cuticle deposition. Pestic. Sci. 1973, 4,737-745. [CrossRef]

42. Gangishetti, U.; Breitenbach, S.; Zander, M.; Saheb, S.K.; Müller, U.; Schwarz, H.; Moussian, B. Effects of benzoylphenylurea on chitin synthesis and orientation in the cuticle of the Drosophila larva. Eur. J. Cell. Biol. 2009, 88, 167-180. [CrossRef] [PubMed]

43. Merzendorfer, H.; Kim, H.S.; Chaudhari, S.S.; Kumari, M.; Specht, C.A.; Butcher, S.; Brown, S.J.; Manak, J.R.; Beeman, R.W.; Kramer, K.J.; et al. Genomic and proteomic studies on the effects of the insect growth regulator diflubenzuron in the model beetle species Tribolium castaneum. Insect Biochem. Mol. Biol. 2012, 42, 264-276. [CrossRef] [PubMed]

44. Su, H.; Gao, Y.; Liu, Y.; Li, X.; Liang, Y.; Dai, X.; Xu, Y.; Zhou, Y.; Wang, H. Comparative transcriptome profiling reveals candidate genes related to insecticide resistance of Glyphodes pyloalis. Bull. Entomol. Res. 2020, 110, 57-67. [CrossRef]

(C) 2020 by the authors. Licensee MDPI, Basel, Switzerland. This article is an open access article distributed under the terms and conditions of the Creative Commons Attribution (CC BY) license (http://creativecommons.org/licenses/by/4.0/). 